\title{
Delaunay Recovery of Cosmic Density and Velocity Probes
}

\author{
W.E. Schaap \& R. van de Weygaert
}

\begin{abstract}
Optimally resolved one-dimensional density and velocity profiles through cosmological N-body simulations are constructed by means of the Voronoi-Delaunay tessellation reconstruction technique. In a fully self-adaptive fashion a strikingly detailed view of the density features and the corresponding cosmic motions is recovered.
\end{abstract}

In essence, N-body simulations of cosmic structure formation are supposed to represent a discrete sampling of underlying continuous density and dynamical fields. The recovery of the corresponding continuous fields is a less than trivial exercise. They are often distorted by manipulated, user-dependent and therefore biased reconstruction schemes. This makes it in particular cumbersome to deal self-consistently with the characteristically multi-scale hierarchical nature of cosmological density fields. As significant is the failure to recover crucial structural aspects of the salient and frequently sharply defined anisotropic - filamentary and wall-like patterns in the cosmic matter distribution.

Recently, Schaap \& van de Weygaert [3] have developed a fully selfadaptive and unbiased method to reconstruct density and related dynamic fields from a discrete and in general nonuniformly sampled set of point locations. It is based on the stochastic geometric concept of Voronoi/Delaunay tessellations and forms an elaboration on the formalism first proposed by Bernardeau \& van de Weygaert [1] for the case of assessing the statistical properties of cosmic velocity fields.

The application of the method to a large $256^{3}$ GIF N-body simulation (LCDM, 141.3 $h^{-1} \mathrm{Mpc}$, courtesy: S. White) [2, 3] provides a beautiful illustration of its sizeable promise. The top panel of fig. 1 presents the particle distribution in a slice through this simulation. The corresponding density field determined through the Delaunay technique is shown in the adjacent panel. Notice how much better than the saturated particle plot this density field manages to elucidate the wealthy and detailed structural features present in this cosmic volume, superbly rendering its high density contrasts.

\footnotetext{
${ }^{1}$ Kapteyn Institute, PO Box 800, 9700 AV Groningen, The Netherlands
} 
Figure 1.1. Slice through a GIF N-body simulation (top), the Delaunay recovered density (center) and density and velocity profiles along the central line (bottom). (We are grateful to S. White for initiating these calculations.)

While the image of the density field already provides evidence of its operation, it is through the objective assessment of the density profile along the central axis of the slice that the success of the method is fully manifested (solid line, lower panel). Evidently, the Delaunay technique yields a faithful representation of the density field over an impressive dynamic range, encompassing gently varying and extended low-density regions as well as the high density contrasts found in compact objects, be it either condensed clumps or the flattened dimension(s) of filaments and walls. Even more compelling is the correlation with the corresponding velocity field along the same line (dashed line). Largely superseding the poor velocity resolution in the conventionally shotnoise-dominated void regions it succeeds in reproducing the matter depleting super-Hubble like peculiar velocity flows (e.g. void at $\approx 123 h^{-1} \mathrm{Mpc}$ ). Even more striking are the sharp velocity transitions encountered at the location of high density peaks, indicating the large induced infall motions along various directions towards these features (e.g. the peak at $\approx 7 h^{-1} \mathrm{Mpc}$ ).

\section{References}

[1] Bernardeau, F., van de Weygaert, R., 1996, MNRAS 279, 693

[2] Kauffmann, G., et al., 1999, MNRAS 303, 188

[3] Schaap, W.E., van de Weygaert, R., 2000, A\&A 363, L29; 2001, in preparation 
This figure "schaap1.gif" is available in "gif" format from: http://arxiv.org/ps/astro-ph/0109261v1 